ISSN 0103-9954

\title{
ESTIMATIVA DOS PARÂMETROS DA FUNÇÃO DE DENSIDADE PROBABILÍSTICA DE WEIBULL POR REGRESSÃO ANINHADA EM POVOAMENTO DESBASTADO DE Pinus taeda L.
}

\section{PARAMETERS ESTIMATION OF A WEIBULL PROBABILISTIC DENSITY FUNCTION BY NEST REGRESSION IN THINNING STAND OF Pinus taeda $L$.}

\author{
Paulo Renato Schneider ${ }^{1}$ César Augusto Guimarães Finger ${ }^{2}$ Luiz Gastão Bernett ${ }^{3}$ \\ Paulo Sérgio Pigatto Schneider ${ }^{4}$ Frederico Dimas Fleig ${ }^{5}$
}

\section{RESUMO}

Este trabalho teve como objetivo estimar os parâmetros da função de densidade probabilística de Weibull para povoamentos desbastados de Pinus taeda, com finalidade de prognosticar as freqüências por unidade de área e classe diamétrica. Os parâmetros foram calculados por regressão aninhada tendo como variáveis independentes a densidade populacional, sítio e idade. O ajuste das funções de densidade probabilística das freqüências por classe diamétrica, no primeiro e segundo desbaste, e no corte raso, apresentou excelente precisão estatística, com boas estimativas de valores da densidade populacional por classe diamétrica. A densidade probabilística e absoluta estimados por classe de diâmetro, para situações distintas de densidades e idade da população, mostrou excelente precisão, com valores estimados muito próximos dos observados.

Palavras-chave: distribuição diamétrica; freqüência; diâmetro; projeção de crescimento.

\begin{abstract}
This work was accomplished with the objective of predicting the parameters of probabilistic density function for thinning stands of Pinus taeda, in the south of Brazil, with the purpose of obtaining the prognosis of the frequencies per unit of area by diameter class. The parameter from Weibull function was estimate through nested regressions with independent variables that express the density of population. Results of the adjustment of the probabilistic density function of the frequencies per diameter class, in the first and second thinning, and cut, presented an excellent statistical precision, with good estimates of values and population density per diameter class. The values prognostic of the probabilistic density per diameter class, considering situations different density and age from the population, showed an excellent precision, with values close to those ones observed.
\end{abstract}

Keywords: diameter distribution; density; diameter; growth prediction.

\section{INTRODUÇÃO}

No manejo florestal, tanto em sistemas com ou sem desbastes, é imprescindível dominar uma metodologia que permita estimativas precisas das densidades populacionais por classe diamétrica, para fins de determinação de sortimentos de madeira para povoamentos, avaliação de estoque, prognose de produção e de avaliações econômicas.

Nos estudos de distribuição de freqüência em populações florestais, têm sido utilizadas funções de distribuição diamétrica, como: Normal, Log-normal, Gamma, Johnson’s SB, Gram-Charlier, Beta e Weibull (BAILEY e DELL, 1973; HAFLEY e SCHREUDER, 1977; MALTAMO et al., 1995; MALTAMO et al., 2000).

A distribuição diamétrica também tem sido descrita pela função Weibull por ser a mais sustentável para estimar as freqüências por classe de diâmetro (GADOW, 1984; BORDERS et al., 1987 ). Assim, na Espanha, a função de Weibull é o modelo mais utilizado para descrever a distribuição diamétrica de florestas

1. Engenheiro Florestal, Dr, Professor Titular do Departamento de Ciências Florestais, Centro de Ciências Rurais, Universidade Federal de Santa Maria, CEP 97105-900, Santa Maria (RS). paulors@smail.ufsm.br.

2. Engenheiro Florestal, Dr, Professor Associado do Departamento de Ciências Florestais, Centro de Ciências Rurais, Universidade Federal de Santa Maria, CEP 97105-900, Santa Maria (RS). finger@smail.ufsm.br

3. Engenheiro Florestal, Técnico da Klabin S.A., CEP 84261-170, Telêmaco Borba, PR. gastao@klabin.com.br

4. Engenheiro Florestal, MSc. pela Universidade Federal de Santa Maria, CEP 97105-900, Santa Maria (RS). psps@mail.ufsm.br

5. Engenheiro Florestal, Dr, Professor Adjunto do Departamento de Ciências Florestais, Centro de Ciências Rurais, Universidade Federal de Santa Maria, CEP 97105-900, Santa Maria, RS. fdfleig@smail.ufsm.br.

Recebido para publicação em 25/03/2008 e aceito em 11/08/2008. 
(ÁLVAREZ, 1997; DEL RIO, 1998; GARCÍA GÜEMES et al., 2002).

Essa função foi desenvolvida por Fischer e Tippert, em 1928, e aplicada por Weibull, em 1939 (PRODAN et al., 1997), tendo sido introduzida com maior ênfase na área florestal por Bailey e Dell (1973). Sua popularidade decorre da relativa simplicidade de uso, da flexibilidade e em conseqüência da facilidade de correlacionar seus parâmetros com algumas características importantes do povoamento.

A caracterização e definição da distribuição diamétrica é fundamental nos estudos de sortimento dos povoamentos, pois aumenta a precisão das estimativas ao longo da existência da floresta, permitindo corrigir os efeitos do manejo e do sítio sobre o estoque ao longo da rotação.

Por causa disso, vários autores justificaram seu uso por considerá-la mais vantajoso que a distribuição Beta. Por essa razão, a função Weibull tem sido extensivamente empregada em estudos de crescimento e produção florestal, com vários trabalhos publicados na literatura nacional e internacional. (SMALLEY E BAILEY (1974), HAFLEY e SCHREUDER (1977), CLUTTER E BELCHER (1978), CLUTTER et al. (1983), LITTLE (1983), BAILEY et al. (1985), ABREU et al. (2000) e NOGUEIRA et al. (2005)), entre outros.

No Brasil, muitos trabalhos de pesquisa sobre distribuição de diâmetros foram desenvolvidos em vários locais e com diferentes espécies, como nos estudos de Couto (1980) para Pinus caribaea var. caribaea, Campos e Turnbull (1981), para Pinus patula, Finger (1982) para Acacia mearnsii, Schneider (1984) para Pinus elliottii, Veiga e Brister (1983) para Pinus elliottii, Batista (1989) para espécies da floresta tropical pluvial.

Segundo Van Laar (1979), nas funções probabilísticas de Weibull, os parâmetros assumem características específicas, sendo "a” um valor da condição de locação da posição inicial da distribuição, com diâmetro mínimo $(a \leq x \prec \infty)$ interpretado como limite inferior do menor centro de classe; "b "um valor de escala (b $<0$ ); e "c" a forma da distribuição de densidade de probabilidade (c $\geq 0$ ), para a condição de $\mathrm{x} \geq 0$.

De acordo com o valor assumido pelos parâmetros o modelo Weibull, pela sua flexibilidade, pode assumir uma ampla variedade de formas de distribuição de freqüência por classe de diâmetro, desde a exponencial, hiperbólica à normal com assimetria positiva ou negativa.

O método para predição dos parâmetros da função de densidade probabilística de Weibull consiste na derivação dos parâmetros partindo das relações entre variáveis associadas ao modelo, sendo esse denominado de Parameter Prediction Model - PPM.

Na maioria dos estudos sobre a projeção do crescimento e produção de povoamentos florestais, os parâmetros são modelados de forma explícita mediante modelos lineares ou não lineares com variáveis preditoras que utilizam parâmetros do povoamento como: densidade, diâmetro médio, altura dominante, índice de sítio e idade. Nesse procedimento de cálculo de parâmetros, embora as relações lineares dos modelos tenham fundamento biológico, em alguns casos, a precisão gerada tem sido baixa (CAO e BURKHART, 1984; CAO, 2004).

Para obtenção dos parâmetros da distribuição de Weibull, pode ser empregado um dos três métodos de ajuste: máxima verossimilhança, descritos em Bailey e Dell (1973), Finger (1982), Gadow (1983); momentos, descritos por Burk e Newberry (1984), Abreu (2000); ou o de percentis utilizado por Bailey e Dell (1973), Cao e Burkhart (1984), Maltamo et al. (2000) e Cao (2004).

O método de máxima verossimilhança necessita de interação para resolução dos estimadores dos parâmetros e utiliza o procedimento interativo de Newton-Raphson, pelo Método dos Mínimos Quadrados Não-Ordinários para regressão não-linear (LEE, 1980).

Mais recentemente Cao (2004) desenvolveu um algoritmo para a solução da função probabilística de Weibull, utilizando o método de estimador de máxima verossimilhança (Maximum Likelihood Estimator MLE - Regression), com os parâmetros calculados para um valor de $a=0,5 . \hat{D}_{o}$ (diâmetro mínimo) e outras duas equações para os parâmetros da escala (b) e forma (c). Para estimativa desses parâmetros, recomenda o ajuste de regressão pelo método dos mínimos quadrados ordinários, tendo como variáveis independentes o espaçamento relativo, altura dominante, número de árvores por hectare e a idade do povoamento.

Cao (2004) desenvolveu ainda um método para ajustar a função de distribuição acumulada (Cumulative Distribuition Function - CDF - Regression) que se baseia no mesmo procedimento anterior, 
exceto que os coeficientes $b_{i}$ e $c_{i}$ são obtidos por cálculo interativo para minimizar a diferença quadrática média entre as freqüências observadas e estimadas.

O procedimento denominado passo invariante baseia-se no princípio de que predições sucessivas, efetuadas da idade $t_{1}$ para $t_{2}$ e da idade $t_{2}$ para $t_{3}$, devem, matematicamente, apresentar as mesmas predições obtidas por um único passo entre as idades $\mathrm{t}_{1}$ e $\mathrm{t}_{3}$ (SOMERS e FARRAR Jr., 1991; SOARES et al., 2007).

Segundo Guimarães (1994), nesse procedimento, as medidas de posição, efetuadas na distribuição em determinada idade, constituem a base da estimativa das magnitudes dessas mesmas medidas em outras idades. Para o ajuste de um modelo de passo invariante, os dados relativos ao número de árvores por classe de diâmetro são ordenados de forma cumulativa, possuindo a classe de maior diâmetro o número total de árvores contidas na parcela.

Na maioria dos trabalhos, foi utilizado o teste de aderência não-paramétrico Kolmogorov-Smirnov, para avaliar a qualidade de ajustamento produzida na distribuição teórica de um conjunto de dados, em razão de sua sensibilidade a qualquer diferença do valor central e da dispersão. O teste representa o grau de concordância entre uma distribuição observada e uma distribuição teórica esperada (GADOW, 1984; CAO, 2004).

Dessa forma, este trabalho teve como objetivo descrever a dinâmica da distribuição diamétrica de florestas de Pinus taeda em diferentes idades, sob influência de desbaste com o emprego da função de densidade probabilística de Weibull.

\section{MATERIAL E MÉTODOS}

Os dados para o presente trabalho foram mensurados em povoamentos de Pinus taeda, localizados

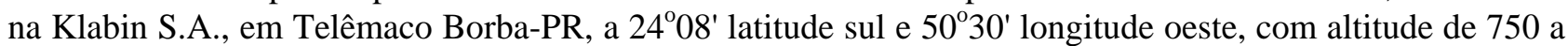
$868 \mathrm{~m}$.

Segundo a classificação de Köppen, o clima é Cfa - Subtropical apresentado temperatura média no mês mais frio: $15,6^{\circ} \mathrm{C}$, temperatura média no mês mais quente: $22,3^{\circ} \mathrm{C}$ e temperatura mínima registrada: $5,2^{\circ} \mathrm{C}$. A precipitação média anual nos últimos 54 anos é 1.508,8 mm com 99,6 dias chuvosos.

O material de origem do solo é predominantemente das formações Rio Bonito, Itararé e Diques de Diabásio. Com base no levantamento e mapeamento de solos os dados foram amostrados em vários sítios, mas especialmene, em solos do tipo Latossolo e Cambissolo, com textura argilosa e média, com vegetação primária de campo e floresta existentes nas áreas de plantio.

\section{Características dos povoamentos}

Os talhões de Pinus taeda amostrados estavam distribuídos sobre unidades de manejo com baixa, média e alta produtividade, definidas em termos de índice de sitio, e foram manejados com objetivo de produção de madeira para serraria, com um e dois desbastes e corte raso aos 20 anos. A densidade inicial foi de 1.600 árvores/ha, com um desbaste aos 9 anos, sistemático da sexta linha e seletivo nas entrelinhas, permanecendo 675 árvores/ha; um segundo desbaste seletivo aos 14 anos, permanecendo 275 árvores/ha.

\section{Amostragem dos dados}

No presente estudo, foi tomado 366 unidades amostrais com dimensão aproximada de $20 \times 30$ metros. No interior de cada unidade amostral foram medidas as circunferências à altura do peito de cada árvore com fita métrica, a altura das vinte primeiras árvores e a altura das árvores dominantes, com o hipsômetro com precisão de décimo de metro.

\section{Distribuição de densidade de probabilidade}

Com o objetivo de gerar os sortimentos para os regimes de manejo dos povoamentos, inicialmente foi necessário conhecer a estrutura de freqüência de indivíduos por classe de diâmetro no tempo. Para isso, foi utilizada a função de densidade probabilística de Weibull com três parâmetros na forma:

$$
f(x)=\left(\frac{c}{b}\right)\left(\frac{x-a}{b}\right)^{c-1} \exp \left[-\left(\frac{x-a}{b}\right)^{c}\right]
$$

Sendo: $\mathrm{x}$ = variável aleatória, no caso o centro de classe de diâmetro; $f(x)=$ freqüência por unidade 
de área; a = parâmetro que indica a locação da posição inicial da distribuição, com diâmetro mínimo $(a \leq x \prec \infty)$ interpretado como limite inferior do menor centro de classe; $\mathrm{b}=$ valor de escala $(\mathrm{b}<0)$; $\mathrm{c}=$ forma da distribuição de densidade de probabilidade (c $>0$ ), para a condição de $\mathrm{x} \geq 0$.

A estimativa dos parâmetros da função de densidade probabilística de Weibull no tempo foi obtida por uma regressão específica para cada parâmetro do modelo de distribuição ajustada pelo método dos mínimos quadrados ordinários, segundo Cao (2004), pela expressão:

$$
y=\exp \left[b_{1}+b_{2} \cdot E+b_{3} \cdot \ln (N)+b_{4} \cdot \ln \left(h_{100}\right)+b_{5} \cdot t^{-1}+\varepsilon\right]
$$

Sendo: $\mathrm{y}=$ um parâmetro específico da função de Weibull; $\mathrm{E}=$ espaçamento relativo, $\left\{\left(10^{4} / N\right)^{0,5} / h_{100}\right\}$, sendo a proporção da distância media entre duas árvores, assumindo espaço quadrático, e a altura dominante; $\mathrm{N}=$ número de árvores por hectare; $\mathrm{h}_{100}=$ altura dominante em metros; $\mathrm{t}$ = idade do povoamento em anos; $\ln =$ logaritmo natural; $\mathrm{b}_{\mathrm{j}}=$ parâmetros; $\varepsilon=$ erro.

Para a solução da função de densidade probabilística de Weibull, foi utilizado o algoritmo de Cao (2004) que otimiza os parâmetros da regressão pela máxima verossimilhança (Maximum Likelihood Estimator - MLE - Regression), no pacote estatístico SAS (Statistical Analysis System).

No ajuste do modelo, o procedimento de Cao (2004) determina as probabilidades de densidade por classe de diâmetro, bastando informar tão somente os diâmetros das unidades amostrais.

Na determinação dos parâmetros foi computado um valor de $a=0,5 . \hat{D}_{o}$ do diâmetro mínimo e duas equações para predizer os parâmetros b e c:

$$
\begin{aligned}
& b=\exp \left[b_{1}+b_{2} \cdot S+b_{3} \cdot \ln (N)+b_{4} \cdot \ln \left(h_{100}\right) b_{5} \cdot t^{-1}\right\rfloor \\
& c=\exp \left[c_{1}+c_{2} \cdot S+c_{3} \cdot \ln (N)+c_{4} \cdot \ln \left(h_{100}\right)+c_{5} \cdot t^{-1}\right]
\end{aligned}
$$

Os coeficientes $b_{1}$ e $c_{1}$ foram obtidos por processo interativo para minimizar a soma do logaritmo da probabilidade de densidade, expresso por:

$$
\sum_{i=1}^{p} \ln \left(L_{i}\right) / n_{i}
$$

Sendo: $\mathrm{p}$ = número de combinações de amostras-idade; $\mathrm{n}_{\mathrm{i}}=$ número de árvores na $\mathrm{i}^{\text {sssima }}$ combinações de amostras-idade (o termo $1 / \mathrm{n}_{\mathrm{i}}$ foi incluído para uma dada equação com número diferente de árvores);

$$
\ln \left(L_{i}\right)=\sum_{j=1}^{n_{i}}\left[\ln (c)-\ln (b)+(c-1) \ln \left(\frac{x_{i j}-a}{b}\right)-\left(\frac{x_{i j}-a}{b}\right)^{c}\right] ; \mathrm{x}_{\mathrm{ij}}=\text { diâmetro da árvore j na i éssima combinações de }
$$
amostras-idade.

Após a obtenção dos parâmetros da função de densidade probabilística de Weibull, o número de árvores em uma dada classe de diâmetro foi estimado por:

$$
n_{i}=N\left\{\exp ^{-\left[\left(\left(d_{i}-w / 2\right)-x_{\min }\right) / b\right] c}-\exp ^{\left[\left(d_{i}+(w / 2)-x_{\min }\right) / b\right] c}\right\}
$$

Sendo: $\mathrm{n}_{\mathrm{i}}=$ número de árvores na classe de diâmetro $\mathrm{i} ; \mathrm{N}=$ tamanho da amostra em número de árvores por hectare; $\mathrm{d}_{\mathrm{i}}=$ diâmetro do centro de classe $\mathrm{i} ; \mathrm{w}=$ amplitude da classe de diâmetro; $\mathrm{x}_{\min }=$ diâmetro mínimo.

A qualidade de ajustamento foi medida pelos índices de aderência, que se referem ao grau de concordância entre uma distribuição observada e uma teórica esperada, a um nível de probabilidade, conforme realizado por CAO, (2004); FINGER, (1982): ANDERSON e DARLING, (1954); pelos testes de:

a) Kolmogorov-Smirnov

$$
K S_{i}=\max \left\{\max _{1 \leq i \leq n_{i}}\left[j / n_{i}\right]-u_{j}\right\},
$$

Sendo: $u_{j}=1-\exp \left\{-\left[\left(x_{j}-a\right) / b\right]^{c}\right\} ; \mathrm{n}_{\mathrm{i}}=$ número de árvores na $i^{\text {th }}$ combinação de idade da amostra; $x_{j}=$ diâmetro, ordenado em ordem ascendente em cada combinação de idade da amostra 
$\left(x_{1} \leq x_{2} \leq \ldots \leq x_{n_{i}}\right)$.

b) Anderson-Darling

$$
A D_{i}=-n_{i}-\sum_{j=1}^{n_{j}}(2 j-1)\left[\ln \left(u_{j}\right)+\left(1-u_{n-j+1}\right) / n_{i}\right]
$$

Sendo: $u_{j}=1-\exp \left\{-\left[\left(x_{j}-a\right) / b\right]\right\} ; \mathrm{n}_{\mathrm{i}}=$ número de árvores na iéssima combinação de idade da amostra; $\mathrm{x}_{\mathrm{j}}=$ diâmetro, ordenado em ordem ascendente em cada combinação de idade da amostra $\left(x_{1} \leq x_{2} \leq \ldots \leq x_{n_{i}}\right)$; a, b, c = parâmetros da distribuição de Weibull.

c) Cramer - von Mises

$$
W=\sum_{i=1}^{n}\left(F(x)-F^{\prime}(x)\right)^{2} \cdot F_{i} \cdot n
$$

Sendo: $F(x)=$ probabilidade de freqüência observada na classe i; $F^{\prime}(x)=$ probabilidade de freqüência estimada na classe $i ; F_{i}=$ probabilidade de freqüência estimada na classe $i ; n=$ freqüência total.

\section{RESULTADOS E DISCUSSÕES}

Os dados amostrados nos povoamentos submetidos a um e dois desbastes e com o corte final para essas duas situações, ajustados pela distribuição de densidade probabilística de Weibull estabeleceram um grupo de regressões que descrevem a dinâmica das freqüências por classe de diâmetro conforme sumarizados na Tabela 1.

TABELA 1: Parâmetros e estatísticas das regressões aninhadas da função Weibull, ajustadas para os desbastes e corte raso.

TABLE 1: Parameters and statistics of the nested regressions of the Weibull function, adjusted for the thinning and clear cut.

\begin{tabular}{cccccc}
\hline $\begin{array}{c}\text { Parâmetros/ } \\
\text { estatísticas }\end{array}$ & $\begin{array}{c}\text { Desbaste } 1^{\mathbf{0}} \\
(10 \text { anos })\end{array}$ & $\begin{array}{c}\text { Desbaste } 2^{\mathrm{o}} \\
(14 \text { anos })\end{array}$ & $\begin{array}{c}\text { Corte raso } \\
\text { desb. }(20 \text { anos })\end{array}$ & $\begin{array}{c}\text { Corte raso } \\
\text { 2 desb. (20 anos) }\end{array}$ \\
\hline $\mathrm{b}_{21}$ & 3,5993 & 4,0952 & 5,7016 & 5,0415 \\
$\mathrm{~b}_{22}$ & $-0,0676$ & $-0,1446$ & $-0,2391$ & $-0,1569$ \\
$\mathrm{~b}_{24}$ & $-3,4766$ & 0,4530 & $-15,6500$ & $-13,0726$ \\
$\mathrm{C}_{21}$ & 3,6594 & $-0,1988$ & 7,8066 & 9,1429 \\
$\mathrm{c}_{22}$ & 0,0134 & 0,1034 & 0,0922 & 0,0605 \\
$\mathrm{C}_{23}$ & $-0,7878$ & $-0,7321$ & $-1,6862$ & $-2,0007$ \\
$\mathrm{C}_{24}$ & $-0,8788$ & 51,3378 & $-24,7994$ & $-19,5106$ \\
\hline SQ-Ponderado & 424,62 & 377,13 & 120,2 & 1022,5 \\
\hline Kolmogorov- & 0,0626 & 0,0438 & 0,0433 & 0,0440 \\
Smirnov & $(\mathrm{p}<0,010)$ & $(\mathrm{p}<0,010)$ & $(\mathrm{p}<0,010)$ & $(\mathrm{p}<0,010)$ \\
\hline Cramer-von & 5,2790 & 1,1588 & 0,8982 & 4,7830 \\
Mises & $(\mathrm{p}<0,005)$ & $(\mathrm{p}<0,005)$ & $(\mathrm{p}<0,005)$ & $(\mathrm{p}<0,005)$ \\
\hline Anderson-Darling & 30,7352 & 7,8186 & 6,1230 & 29,5157 \\
& $(\mathrm{p}<0,005)$ & $(\mathrm{p}<0,005)$ & $(\mathrm{p}<0,005)$ & $(\mathrm{p}<0,005)$ \\
\hline Desvio Padrão & 4,0921 & 4,0921 & 6,1708 & 5,8461 \\
\hline Assimetria & 0,4208 & 0,4208 & 0,4033 & 0,4361 \\
\hline Curtose & 0,3192 & 0,3192 & $-0,0856$ & 0,3135 \\
\hline
\end{tabular}

Essas equações forneceram a flexibilidade e dinâmica necessária à distribuição de freqüência por classe de diâmetro, em decorrência da variação da idade $(\mathrm{t})$, altura dominante $\left(\mathrm{h}_{100}\right)$ e da densidade do povoamento expressa pelo número de árvores por hectare $(\mathrm{N})$ :

$$
\begin{aligned}
& b=\exp \left(\mathrm{b}_{21}+\mathrm{b}_{22} \cdot \ln \mathrm{N}+\mathrm{b}_{23} \cdot 1 / \mathrm{t}\right) \\
& c=\exp \left(\mathrm{c}_{21}+\mathrm{c}_{22} \cdot \ln \mathrm{N}+\mathrm{c}_{23} \cdot \operatorname{lnh} \mathrm{h}_{100}+\mathrm{c}_{24} \cdot 1 / \mathrm{t}\right)
\end{aligned}
$$


As estatísticas relacionadas na Tabela 1, determinadas para o primeiro e segundo desbastes e corte raso com um de dois desbastes mostram, tanto para o teste de Kolmogorov-Smirnov, como para o de Cramer-von Mises e Anderson-Darling, valores não-significativos de assimetria e curtose indicando que os dados apresentaram distribuição normal.

Na Tabela 2, os valores observados e estimados em percentis para o primeiro e segundo desbastes e corte final em povoamentos com um e dois desbastes apresentam somente pequenas diferenças entre os valores observados e estimados indicando a boa precisão do método.

Esses percentis da freqüência por classe de diâmetro, do primeiro e segundo desbastes realizados aos 10 e 14 anos respectivamente foram representados de forma gráfica nas Figuras 1 e 2, e para o corte raso aos 20 anos, precedidos de um e dois desbastes, nas Figura 3 e 4 respectivamente. Nestas, foi possível observar a mudança na forma das distribuições de freqüência quando da variação dos valores do diâmetro mínimo, médio e máximo ocorrido nos momentos de desbastes e corte raso. Tais mudanças são normais no manejo e se devem à dinâmica da densidade populacional no tempo, o que pode ser constatado pelos valores diferentes de assimetria e curtose obtidos para os desbastes e corte raso.

TABELA 2: Percentis da distribuição de Weibull para os desbastes e corte raso com um e dois desbastes. TABLE 2: Percents of the Weibull distribution for thinning and cut with one and two thinning.

\begin{tabular}{c|c|c|c|c|c|c|c|c}
\hline \multirow{2}{*}{ Percentil } & \multicolumn{2}{|c|}{$\begin{array}{c}\text { Desbaste } \\
1^{\circ}(10 \text { anos })\end{array}$} & \multicolumn{2}{c|}{$\begin{array}{c}\text { Desbaste } \\
2^{\circ}(14 \text { anos })\end{array}$} & \multicolumn{2}{c|}{$\begin{array}{c}\text { Corte raso com } 1 \\
\text { desb.(20 anos) }\end{array}$} & \multicolumn{2}{c}{$\begin{array}{c}\text { Corte raso com } \\
2 \text { desb.(20 anos) }\end{array}$} \\
\cline { 2 - 9 } & Obs. & Est. & Obs. & Est. & Obs. & Est. & Obs. & Est. \\
\hline 1,0 & 8,91000 & 6,29807 & 17,8300 & 13,7546 & 16,8700 & 11,7509 & 21,0100 & 16,1777 \\
5,0 & 11,14000 & 9,35470 & 20,0500 & 17,6782 & 18,7800 & 16,4968 & 24,5100 & 21,4090 \\
10,0 & 12,41000 & 11,14066 & 21,0100 & 19,7502 & 20,3700 & 19,1630 & 26,1000 & 24,2290 \\
25,0 & 14,32000 & 14,21666 & 23,2400 & 23,0536 & 23,5500 & 23,6187 & 29,2800 & 28,7953 \\
50,0 & 17,19000 & 17,59943 & 25,7800 & 26,3963 & 27,6900 & 28,3623 & 32,7900 & 33,4943 \\
75,0 & 20,05000 & 20,82414 & 28,6500 & 29,3692 & 32,4700 & 32,7637 & 36,6100 & 37,7326 \\
90,0 & 23,55000 & 23,55346 & 31,5100 & 31,7556 & 36,2900 & 36,4130 & 41,0600 & 41,1714 \\
95,0 & 25,46000 & 25,10703 & 33,4200 & 33,0687 & 38,8300 & 38,4629 & 43,6100 & 43,0766 \\
99,0 & 29,60000 & 27,86911 & 37,2400 & 35,3322 & 43,6100 & 42,0638 & 49,0200 & 46,3811 \\
\hline
\end{tabular}

Em que: Obs. = observado; Est. = estimado.

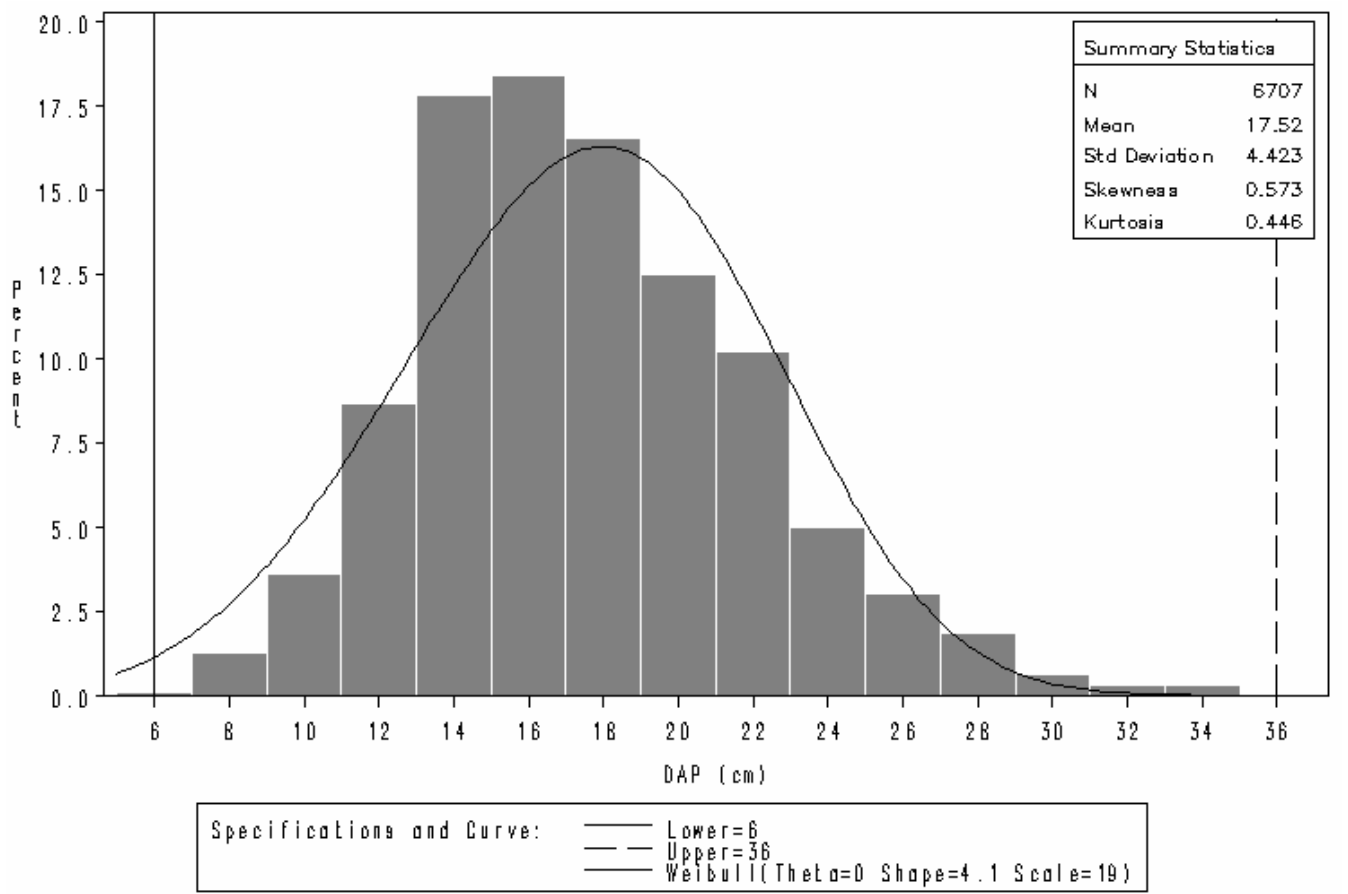

FIGURA 1: Percentis das freqüências de árvores por classe diamétrica do primeiro desbaste.

FIGURE 1: Percents of the frequencies of trees per diameter class of the first thinning. 


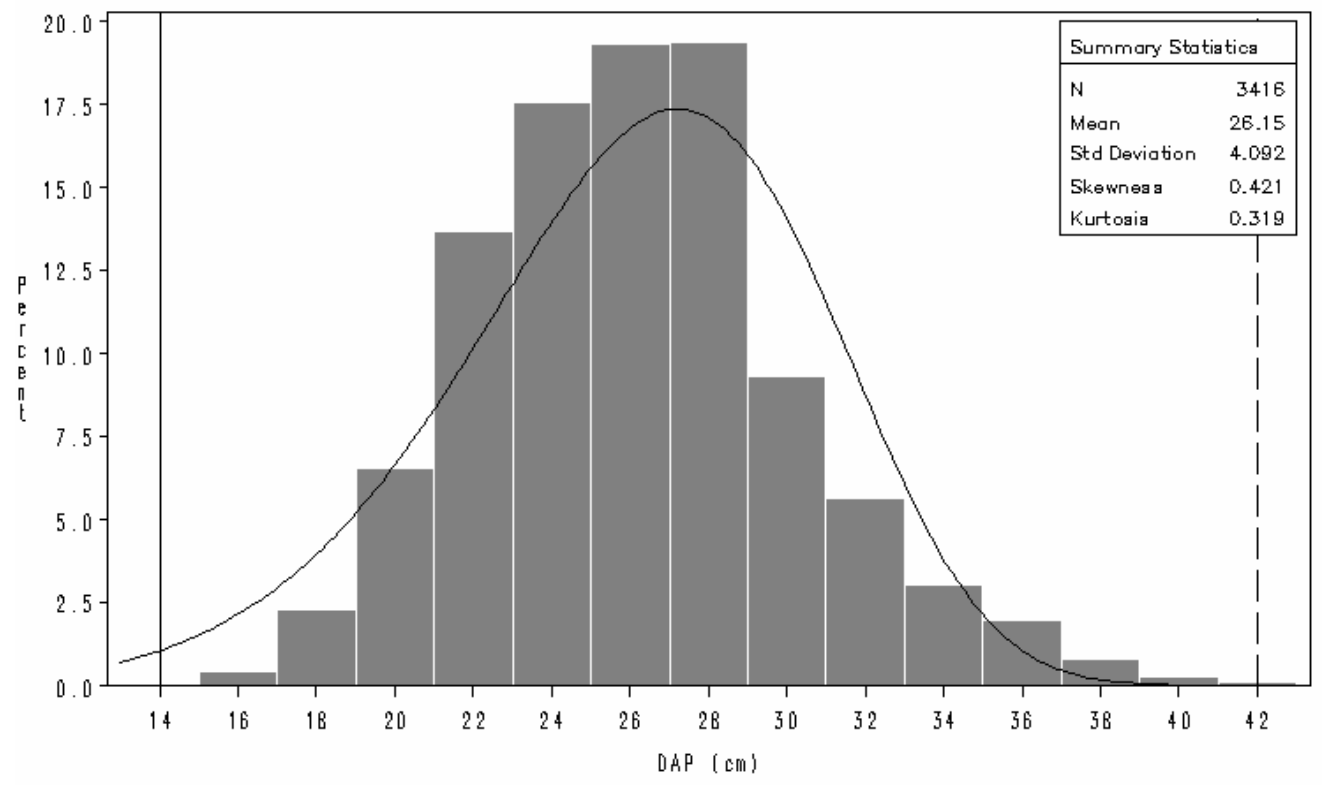

Specifications and Curve: - Lower=14

Weper=42

FIGURA 2: Percentis das freqüências de árvores por classe diamétrica do segundo desbaste.

FIGURE 2: Percents of the frequencies of trees per diameter class of the second thinning.

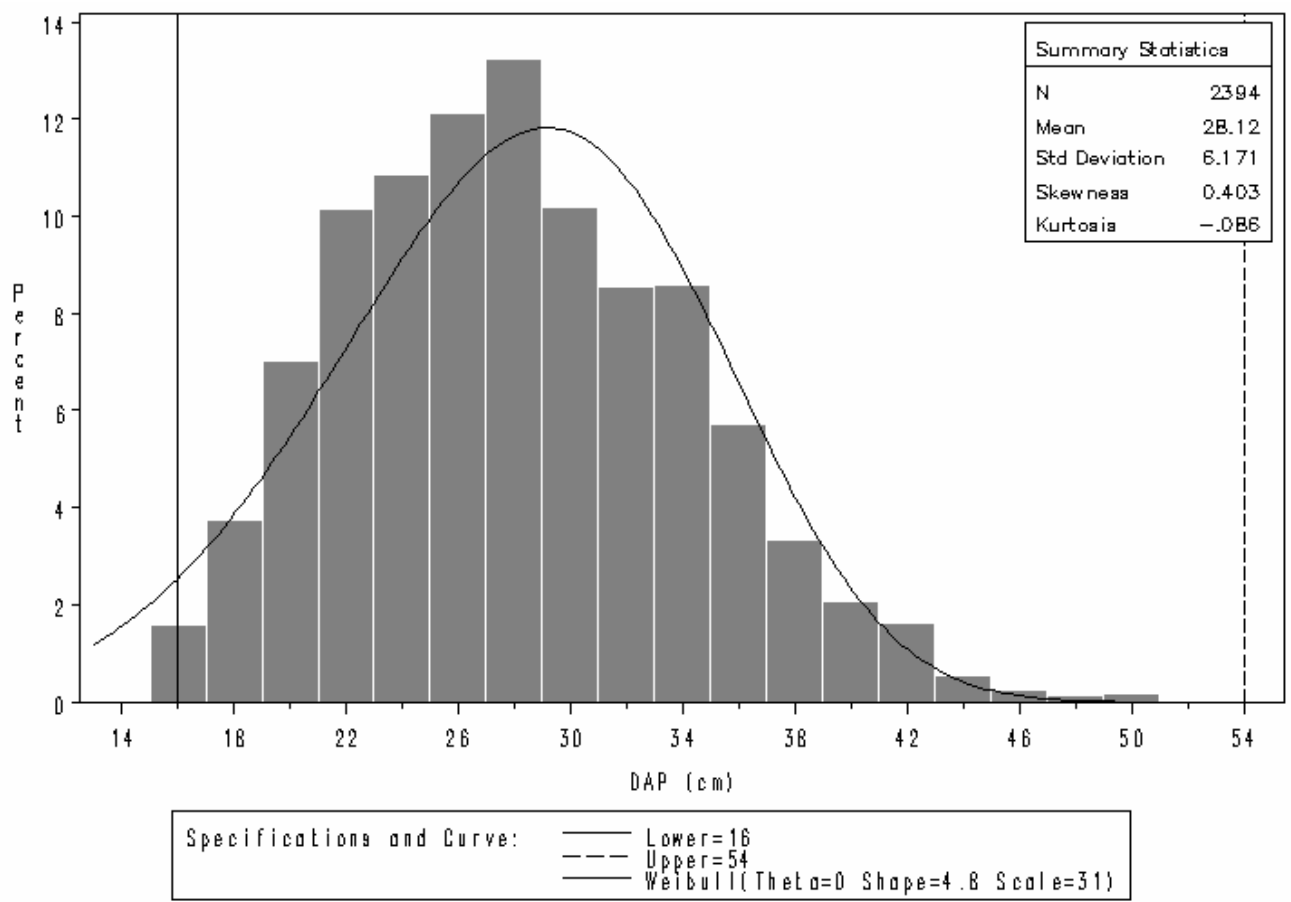

FIGURA 3: Percentis das freqüências de árvores por classe diamétrica do corte raso com um desbaste. FIGURE 3: Percents of the frequencies of trees per diameter class of the cut with one thinning. 


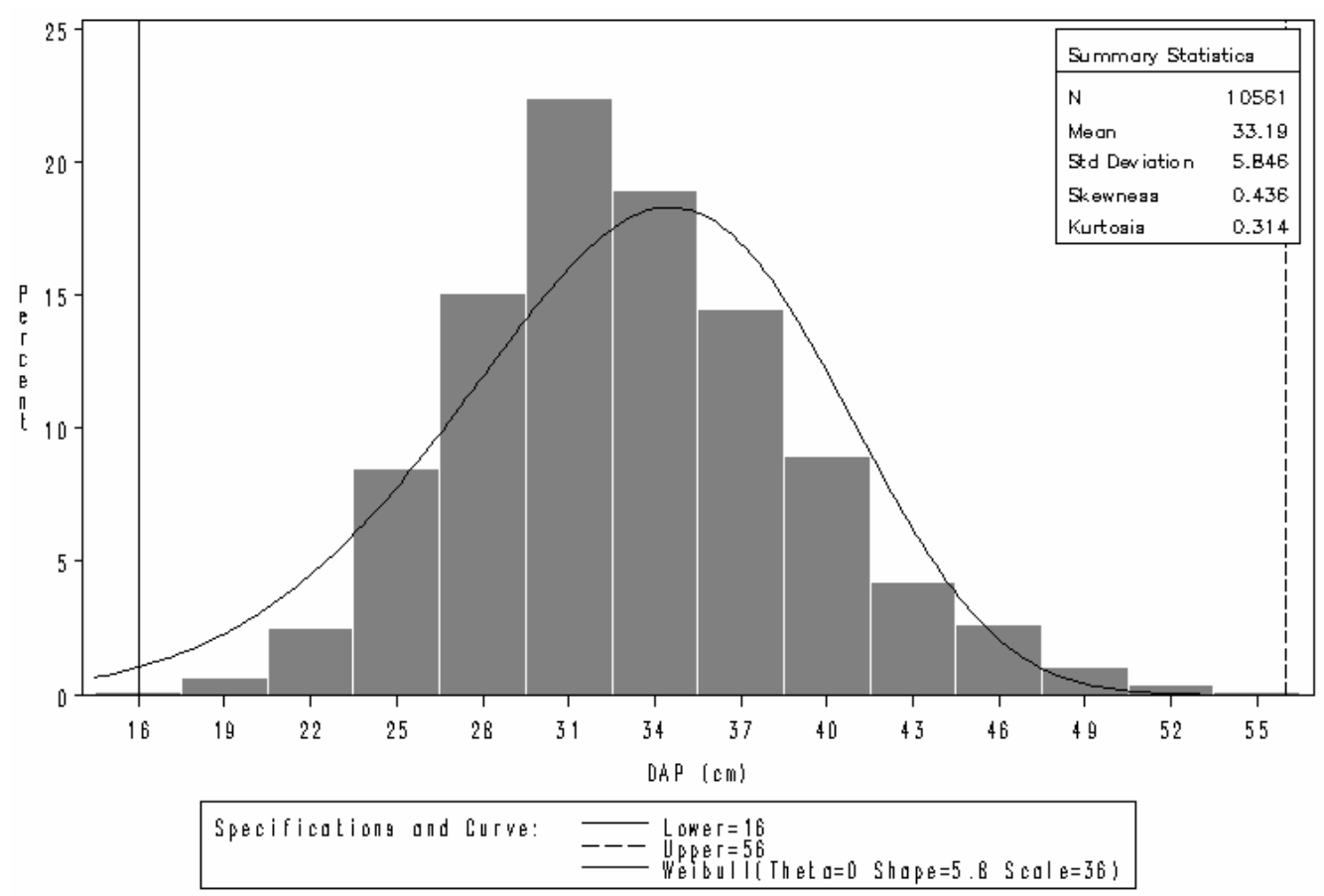

FIGURA 4: Percentis das freqüências de árvores por classe diamétrica do corte raso com dois desbaste.

FIGURE 4: Percents of the frequencies of trees per diameter class of the cut of two thinning.

A posterior estimativa dos parâmetros b e $\mathbf{c}$ da distribuição de freqüência de Weibull, com regressões aninhadas, considerando os dados em conjunto único, independente do número de intervenções realizadas, resultaram na menor soma de quadrados ponderados, conforme a Tabela 3. Observou-se que todos os valores estatísticos dos parâmetros estimados tiveram um erro-padrão baixo, inferior a 3,13, indicando o bom ajuste e precisão e comprovando a eficiência da metodologia empregada.

TABELA 3: Parâmetros e estatísticas das equações aninhadas dos parâmetros b e c da distribuição de Weibull com dados agrupados.

TABELE 3: Parameters and statistics of the next equations of the parameters $\mathbf{b}$ and $\mathbf{c}$ of the distribution of Weibull with contained date.

\begin{tabular}{|c|c|c|c|c|}
\hline \multirow[t]{2}{*}{ Parâmetros } & \multirow{2}{*}{$\begin{array}{l}\text { Valore dos } \\
\text { parâmetros }\end{array}$} & \multirow[t]{2}{*}{ Erro-padrão } & \multicolumn{2}{|c|}{ Limites de confiança (95\%) } \\
\hline & & & Inferior & Superior \\
\hline $\mathrm{b}_{11}$ & 5,3699 & 0,0589 & 5,2544 & 5,4853 \\
\hline $\mathrm{B}_{12}$ & $-0,2131$ & 0,0109 & $-0,2344$ & $-0,1918$ \\
\hline $\mathrm{B}_{13}$ & $-12,0091$ & 0,6803 & $-13,3425$ & $-10,6756$ \\
\hline $\mathrm{C}_{21}$ & 7,2352 & 0,9949 & 5,2852 & 9,1853 \\
\hline $\mathrm{C}_{22}$ & $-0,2550$ & 0,0438 & $-0,3409$ & $-0,1691$ \\
\hline $\mathrm{C}_{23}$ & $-0,9947$ & 0,2503 & $-1,4854$ & $-0,5041$ \\
\hline $\mathrm{C}_{24}$ & $-10,1049$ & 3,1356 & $-16,2510$ & $-3,9588$ \\
\hline Testes & \multicolumn{2}{|c|}{ Estatísticas } & \multicolumn{2}{|c|}{ Valor de $\mathrm{p}$} \\
\hline $\begin{array}{c}\text { Kolmogorov- } \\
\text { Smirnov }\end{array}$ & $\mathrm{D}$ & 0,02554 & Prob > D & $<0,010$ \\
\hline Cramer-von Mises & W-Sq & 1,92933 & Prob $>$ W-Sq & $<0,005$ \\
\hline Anderson-Darling & $\mathrm{A}-\mathrm{Sq}$ & 11,24345 & Prob $>$ A-Sq & $<0,005$ \\
\hline
\end{tabular}

O conjunto de 22.139 dados de diâmetros utilizados apresentaram média de 35,06 cm, com um desvio-padrão de 7,08, assimetria de 0,15 e curtose de 0,1016. Os testes de normalidade de KolmogorovSmirnov, como de Cramer-von Mises e Anderson-Darling, indicam a existência de distribuição normal, comprovado pela baixa probabilidade de significância dos parâmetros estatísticos determinados.

Os parâmetros b e c estimados com dados agrupados permitiram gerar os percentis e, posteriormente, a densidade absoluta de árvores por classe de diâmetro. A prognose das freqüências por hectare distribuídas 
em classes de diâmetro para uma situação de manejo com desbastes nas idades de 10 e 14 anos e corte raso aos 20 anos, considerando ainda os demais parâmetros relacionados na Tabela 4. O resultado demonstra a eficiência das funções para b e c calculadas com dados agrupados cujos resultados encontram-se na Tabela 5.

TABELA 4: Parâmetros dendrométricos do primeiro e segundo desbastes e do corte raso.

TABLE 4: Dendrometric parameters of the first and second thinning and at clear cut age.

\begin{tabular}{l|c|c|c}
\hline Momento da operação & $\begin{array}{c}\text { Idade } \\
\text { (anos) }\end{array}$ & $\begin{array}{c}\mathrm{h}_{100} \\
(\mathrm{~m})\end{array}$ & $\begin{array}{c}\mathrm{N} \\
\text { (ha) }\end{array}$ \\
\hline Remanescente $1^{\circ}$ desbaste & 10 & 14 & 675 \\
Remanescente $2^{\circ}$ desbaste & 14 & 20 & 300 \\
Corte Raso & 20 & 26 & 300 \\
\hline
\end{tabular}

TABELA 5: Densidades populacionais remanescentes prognosticadas no primeiro e segundo desbastes e no corte raso.

TABLE 5: Predicted remained density population of the first and second thinning and at clear cut age.

\begin{tabular}{c|ccc}
\hline Centro classe & \multicolumn{3}{|c}{ Densidade populacional (N/ha) } \\
\cline { 2 - 4 } DAP $(\mathrm{cm})$ & Remanescente $(10$ anos) & Remanescente $(14$ anos) & Corte raso (20 anos) \\
\hline 8 & 0,04 & - & - \\
10 & 0,66 & - & - \\
12 & 4,45 & 0,02 & - \\
14 & 18,79 & 0,09 & 0,02 \\
16 & 57,23 & 0,35 & 0,06 \\
18 & 129,36 & 1,10 & 0,19 \\
20 & 202,14 & 2,95 & 0,49 \\
22 & 183,06 & 6,92 & 1,11 \\
24 & 71,41 & 14,44 & 2,30 \\
26 & 7,74 & 26,84 & 4,41 \\
28 & 0,12 & 43,65 & 7,88 \\
30 & - & 59,75 & 13,19 \\
32 & - & 64,45 & 20,63 \\
34 & - & 49,56 & 29,89 \\
36 & - & 23,58 & 39,57 \\
38 & - & 5,72 & 46,79 \\
40 & - & 0,55 & 47,87 \\
42 & - & 0,01 & 40,59 \\
44 & - & - & 26,95 \\
46 & - & - & 13,04 \\
48 & - & - & 4,21 \\
50 & - & - & 0,81 \\
\hline
\end{tabular}

A forma gráfica desses resultados, apresentada na Figura 6, permite uma melhor visualização da dinâmica da distribuição, em que pode ser verificada a variação dos diâmetros mínimo, médio e máximo, e suas freqüências nas situações de manejo propostas para o Pinus taeda.

A simulação continua do manejo dos povoamentos apoiada por critérios de decisão técnica e econômica permitirá a otimização da produção florestal. 


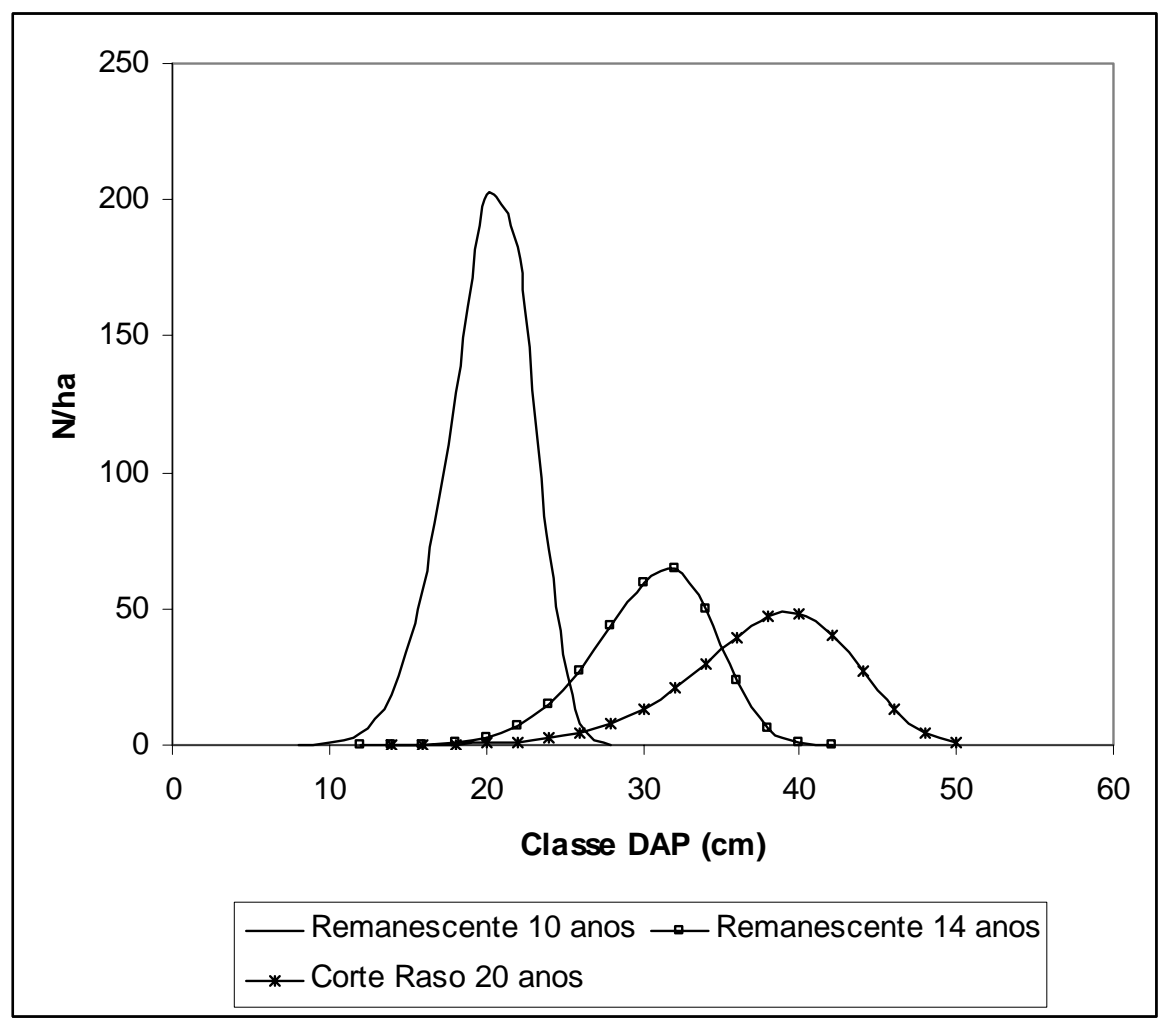

FIGURA 6: Prognose das densidades de árvores por classe diamétrica para desbastes e corte raso. FIGURE 6: Density of trees predict per diameter class for thinnings and cut.

\section{CONCLUSÕES}

Os resultados obtidos no presente trabalho permitem concluir que a distribuição diamétrica e sua dinâmica no tempo para diferentes situações de manejo podem ser descritas, com alta precisão, pela função de densidade probabilística de Weibull, com parâmetros estabelecidos em função de variáveis de fácil determinação, como a altura dominante, número de árvores por hectare e idade, utilizando o método de regressão aninhada.

\section{REFERÊNCIAS BIBLIOGRÁFICAS}

ABREU, E. Modelagem da prognose precoce do volume por classe diamétrica para Eucalyptus grandis. Lavras, 2000. 70 f. Tese (Mestrado em Manejo Florestal) - Universidade Federal de Lavras, Lavras.

ÁLVAREZ, J. G. Análisis y caracterización de las distribuciones diamétricas de Pinus pinaster Ait. en Galicia. 1997. 269 p. Tesis Doctoral, ETSIM-UPM, Madrid.

ANDERSON, T. W.; DARLING, D.A. A test of goodness of fit. Jor. Am. Stat. Assoc. v. 49, p. 765-769. 1954.

BAILEY, R. L. et al. A compatible model relating slash pine plantation survival to density, age, site index and type and intensity of thinning. Forest Science, v. 31, n. 1, p. 181-189, 1985.

BAILEY, R. L.; DELL, T. R. Quantifying diameter distribution with the Weibull-function. For. Sci., v. 19, p. 97 - 104. 1973.

BATISTA, J. L. F. A função Weibull como modelo para a distribuição de diâmetro de espécies arbóreas tropicais., 1989. 125 f. Dissertação (Mestrado) - Escola Superior de Agricultura "Luiz de Queiroz", Piracicaba.

BORDERS, B. E; SOUTER, R. A.; BAILEY, R. L.; WARE, K. D. Percentile-based distributions characterize forest stand tables. Forest Science, v. 33, p. 570 - 576, 1987.

BURK, T.; NEWBERRY, J. A simple algorithm for moment-based recovery of Weibull distribution parameters. Forest Science, v. 30, n. 2, p. 329 - 332, 1984.

CAMPOS, J. C. C.; TURNBULL, K. Um sistema para estimar a produção por classe de diâmetro e sua aplicação na interpretação do efeito de desbaste. Revista Árvore, Viçosa, v. 5, n. 1, p. l-16, 1981.

CAO, Q. V. Predicting parameters of a Weibull function for modeling diameter distribution. Forest Science, v. 50, n. 4, p.682-685, 2004. 
CAO, Q.; BURKHART, H. A segmented distribution approach for modeling diameter frequency data. Forest Science, v. 30, n. 1. p. $129-137,1984$.

CLUTTER, J .L. et al. Timber management: a quantitative approach. New York: John Wiley \& Sons, Inc., 1983. 333 p.

CLUTTER, J. L.; BELCHER, D. M. Yield of site repaired slash pine plantations in the lower coastal, plain of Georgia and Florida. In: GROWTH MODELS FOR LONG-TERM FORECASTING OF TIMBER YIELDS, 1978, Blacksburg. Proceedings... Blacksburg: IUFRO, School of Forestry and Wildlife Resources, State Unversity, 1978. p. 53-70.

COUTO, H. T. Z. Distribuições de diâmetros em plantações de Pinus caribaea Morelet var. caribaea., 1980.83 p. Tese (Livre-Docência) - Escola Superior de Agricultura “Luiz de Queiroz”, Piracicaba.

DEL RIO, M. Régimen de claras y modelo de producción para Pinus sylvestris L. en los sistemas Central Ibérico. 1998. 219 p. Tesis Doctoral, ETSIM-UPM, Madrid.

FINGER, C. A. G. Distribuição de diâmetros em acácia negra, Acacia mearnsii de Wild em diferentes povoamentos e idades. 1982. 124 f. Dissertação (Mestrado em Ciências Florestais) - Universidade Federal do Paraná, Curitiba.

GADOW, K. Die Erfassung von Durchmesserverteilungen in gleichaltigen Kiefernbeständen. Forstweiss. Centralbl. v. 103, p. 369 - 374, 1984.

GARCÍA GÜEMES, C.; CAÑADAS, N.; MONTERO, G. Modelización de la distribución diamétrica de las masas de Pinus pinea L. de Valladolid (España) mediante la function Weibull. Invest. Agr. Sist. Recur. For. v. 11, p. 263 - 282, 2002.

GUIMARÃES, D. P. Desenvolvimento de um modelo de distribuição diamétrica de passo invariante para prognose e projeção da estrutura de povoamentos de eucalipto. 1994. 160 f. Tese (Doutorado em Ciência Florestal) - Universidade Federal de Viçosa, Viçosa.

HAFLEY, W. L.; SCHREUDER, H. T. Statistical distributions for fitting diameter and height data in even-aged stands. Can. J. For. Res., v. 4, p. 481 - 487, 1977.

KLABIN FLORESTAL PARANÁ, Plano de manejo florestal. Telêmaco Borba, 2002, 149 p.

LEE, E. T. Statistical methods for survival data analysis. Belmont: Lifetime Learning, 1980. 557 p.

LITTLE, S. N. Weibull diameter distributions for mixed stands of western conifers. Canadian Journal of Forest Research, v. 13, n. 1, p. 85-88, 1983.

MALTAMO, M. et al. Comparison of percentile based prediction on the Weibull distribution in describing the diameter distribution of heterogeneous Scots pine stands. Forest Ecology and Managment., v 133, p. 263-274, 2000.

MALTAMO, M.; PUUMALAINEN, J.; PÄIVINEN, R. Comparison of Beta and Weibull functions for modeling basal area diameter distribution in stands of Pinus sylvestris and Picea abies. Scand. Jor. For. Res., v. 10, p. 284-295, 1995. NOGUEIRA, G. S. et al. Modelo de distribuição diamétrica para povoamentos de Eucalyptus sp. submetidos a desbaste. Revista Árvore, v. 29, n. 4, p. 579-589, 2005.

PRODAN, M.; PETERS, R.; COX, F., et al. Mensura Forestal. San José: GTZ, 1997. 561 p.

SCHNEIDER, P. R. Betriebswirtschaftliche und Ertragskundliche Grundlagen der Forsteinrichtung in Südbrasilien am Beispiel von Pinus elliottii. Freiburg, Diss, Der Albert-Ludwigs-Uversität, Freiburg, 1984,192 p. (Tese de Doutorado).

SMALLEY, G. W.; BAILEY, R. L. Yield tables and stand structure for loblolly pine plantations in Tennessee, Alabama and Georgia highlands. Washington: USDA, 1974. 81 p. (Forest Service Research Paper SO, 96).

SOARES, S. T. et al. Avaliação de um modelo de passo invariante na predição da estrutura de um povoamento de Eucalyptus sp. Revista Árvore, v. 31, n. 2, p. 275-283. 2007.

SOMERS, G. L.; FARRAR Jr., R. M. Biomathematical growth equations for natural longleaf pine stands. Forest Science, v. 37, n. 1, p. 227-244, 1991.

VEIGA, R. A. A.; BRISTER, G. H. Avaliação de funções de rendimento para plantações de Pinus elliottii no sudeste dos Estados Unidos da América. Silvicultura. São Paulo, v. 8, n. 28, p.715-719, 1983.

VAN LAAR, A. Biometrische Methoden in der Forstwissenschaft. Teil I: Verfahrensgrundlagen. South Africa: University of Stellenbosch, 1979. 385 p. 